\title{
PRIME TWISTS OF ELLIPTIC CURVES
}

\author{
DANIEL KRIZ AND CHAO LI
}

\begin{abstract}
For certain elliptic curves $E / \mathbb{Q}$ with $E(\mathbb{Q})[2]=\mathbb{Z} / 2 \mathbb{Z}$, we prove a criterion for prime twists of $E$ to have analytic rank 0 or 1 , based on a mod 4 congruence of 2-adic logarithms of Heegner points. As an application, we prove new cases of Silverman's conjecture that there exists a positive proposition of prime twists of $E$ of rank zero (resp. positive rank).
\end{abstract}

\section{INTRODUCTION}

1.1. Silverman's conjecture. Let $E / \mathbb{Q}$ be an elliptic curve. For a square-free integer $d$, we denote by $E^{(d)} / \mathbb{Q}$ its quadratic twist by $\mathbb{Q}(\sqrt{d})$. Silverman made the following conjecture concerning the prime twists of $E$ (see [OS98, p.653], [0no97, p.350]).

Conjecture 1.1 (Silverman). Let $E / \mathbb{Q}$ be an elliptic curve. Then there exists a positive proportion of primes $\ell$ such that $E^{(\ell)}$ or $E^{(-\ell)}$ has rank $r=0$ (resp. $r>0$ ).

Remark 1.2. Conjecture 1.1 is known for the congruent number curve $E: y^{2}=x^{3}-x$. In fact, $E^{(\ell)}$ has $\operatorname{rank} r=0$ if $\ell \equiv 3(\bmod 8)$ and $r=1$ if $\ell \equiv 5,7(\bmod 8)$. This follows from classical 2-descent for $r=0$ and Birch [Bir70] and Monsky [Mon90] for $r=1$ (see also [Ste75]).

Remark 1.3. Although Conjecture 1.1 is still open in general, many special cases have been proved. For $r=0$, see Ono [Ono97] and Ono-Skinner [OS98, Cor. 2] (including all elliptic curves with conductor $\leq 100)$. For $r=1$, see Coates-Y. Li-Tian-Zhai [CLTZ15, Thm. 1.1].

In our recent work [KL16, Thm. 3.3], we have proved Conjecture 1.1 (for both $r=0$ and $r=1$ ) for a wide class of elliptic curves with $E(\mathbb{Q})[2]=0$. The goal of this short note is to extend our method to certain elliptic curves with $E(\mathbb{Q})[2] \cong \mathbb{Z} / 2 \mathbb{Z}$.

1.2. Main results. Let $E / \mathbb{Q}$ be an elliptic curve of conductor $N$. We will use $K$ to denote an imaginary quadratic field satisfying the Heegner hypothesis for $N$ :

each prime factor $\ell$ of $N$ is split in $K$.

We denote by $P \in E(K)$ the corresponding Heegner point, defined up to sign and torsion with respect to a fixed modular parametrization $\pi_{E}: X_{0}(N) \rightarrow E$. Let

$$
f(q)=\sum_{n=1}^{\infty} a_{n}(E) q^{n} \in S_{2}^{\text {new }}\left(\Gamma_{0}(N)\right)
$$

be the normalized newform associated to $E$. Let $\omega_{E} \in \Omega_{E / \mathbb{Q}}^{1}:=H^{0}\left(E / \mathbb{Q}, \Omega^{1}\right)$ such that

$$
\pi_{E}^{*}\left(\omega_{E}\right)=f(q) \cdot d q / q
$$

Date: November 29, 2017.

2010 Mathematics Subject Classification. 11G05 (primary), 11G40 (secondary).

Key words and phrases. elliptic curves, quadratic twists, Heegner points, Silverman's conjecture. 
We denote by $\log _{\omega_{E}}$ the formal logarithm associated to $\omega_{E}$.

Our main result is the following criterion for prime twists of $E$ of analytic (and hence algebraic) rank 0 or 1.

Theorem 1.4. Let $E / \mathbb{Q}$ be an elliptic curve. Assume $E(\mathbb{Q})[2] \cong \mathbb{Z} / 2 \mathbb{Z}$ and $E$ has no rational cyclic 4-isogeny. Assume there exists an imaginary quadratic field $K$ satisfying the Heegner hypothesis for $N$ such that

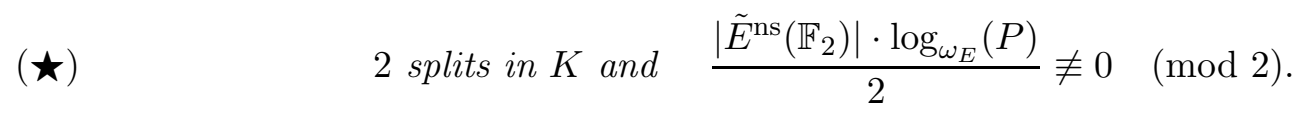

Let $\mathcal{S}$ be the set of primes

$$
\mathcal{S}:=\left\{\ell \nmid 2 N: \ell \text { splits in } K,\left|E\left(\mathbb{F}_{\ell}\right)\right| \not \equiv 0 \bmod 4\right\} .
$$

Let $\mathcal{N}$ be the set of signed primes

$$
\mathcal{N}=\{d= \pm \ell: \ell \in \mathcal{S}, \text { any odd prime } q \| N \text { splits in } \mathbb{Q}(\sqrt{d})\} .
$$

Then for any $d \in \mathcal{N}$, we have the analytic rank $r_{\text {an }}\left(E^{(d)} / K\right)=1$. In particular,

$$
r_{\text {an }}\left(E^{(d)} / \mathbb{Q}\right)= \begin{cases}0, & \text { if } w\left(E^{(d)} / \mathbb{Q}\right)=+1, \\ 1, & \text { if } w\left(E^{(d)} / \mathbb{Q}\right)=-1 .\end{cases}
$$

where $w\left(E^{(d)} / \mathbb{Q}\right)$ denotes the global root number of $E^{(d)} / \mathbb{Q}$.

Remark 1.5. Recall that $\left|\tilde{E}^{\mathrm{ns}}\left(\mathbb{F}_{\ell}\right)\right|$ denotes the number of $\mathbb{F}_{\ell}$-points of the nonsingular part of the $\bmod \ell$ reduction of $E$, which is $\left|E\left(\mathbb{F}_{\ell}\right)\right|=\ell+1-a_{\ell}(E)$ if $\ell \nmid N, \ell \pm 1$ if $\ell|| N$ and $\ell$ if $\ell^{2} \mid N$.

Remark 1.6. The assumption on Heegner points in Theorem 1.4 forces $r_{\text {an }}(E / \mathbb{Q}) \leq 1$.

As a consequence, we deduce the following cases of Silverman's conjecture.

Theorem 1.7. Let $E / \mathbb{Q}$ as in Theorem 1.4. Let $\phi: E \rightarrow E_{0}:=E / E(\mathbb{Q})[2]$ be the natural 2-isogeny. Assume the fields $\mathbb{Q}\left(E[2], E_{0}[2]\right), \mathbb{Q}(\sqrt{-N}), \mathbb{Q}(\sqrt{q})$ (where $q$ runs over odd primes $q \| N$ ) are linearly disjoint. Then Conjecture 1.1 holds for $E / \mathbb{Q}$.

1.3. Novelty of the proof. The proof of [KL16, Thm. 3.3] mentioned above uses the mod 2 congruence between 2-adic logarithms of Heegner points on $E$ and $E^{(d)}$ (recalled in $\$ 3.1$ below), arising from the isomorphism of Galois representations $E[2] \cong E^{(d)}[2]$. For the congruence to be nontrivial on both sides, one needs the extra factor $\left|E\left(\mathbb{F}_{\ell}\right)\right|$ appearing in the formula to be odd for $\ell \mid d$. This is only possible when $E(\mathbb{Q})[2]=0$.

When $E(\mathbb{Q})[2] \neq 0$, we instead take advantage of the exceptional isomorphism between the mod 4 semisimplified Galois representations $E[4]^{\mathrm{ss}} \cong E^{(d)}[4]^{\mathrm{ss}}$, and consequently a $\bmod 4$ congruence between 2-adic logarithm of Heegner points. When $E(\mathbb{Q})[2]=\mathbb{Z} / 2 \mathbb{Z}$ and $E$ has no rational cyclic 4-isogeny, it is possible that the extra factor $\left|E\left(\mathbb{F}_{\ell}\right)\right|$ is even but nonzero $\bmod 4$. This is the key observation to prove Theorem [1.4. The application Theorem 1.7 then follows by Chebotarev's density after translating the condition $\left|E\left(\mathbb{F}_{\ell}\right)\right| \not \equiv 0(\bmod 4)$ into an inert condition for $\ell$ in $\mathbb{Q}(E[2])$ and $\mathbb{Q}\left(E_{0}[2]\right)$ (Lemma 4.1).

1.4. Acknowledgments. The examples in this note are computed using Sage ([Sag16 $])$. 


\section{EXAmples}

Let us illustrate the main results by two explicit examples.

Example 2.1. Consider the elliptic curve (in Cremona's labeling)

$$
E=256 b 1: y^{2}=x^{3}-2 x
$$

with $E(\mathbb{Q})[2] \cong \mathbb{Z} / 2 \mathbb{Z}$. It has $j$-invariant 1728 and $C M$ by $\mathbb{Q}(i)$. The imaginary quadratic field $K=\mathbb{Q}(\sqrt{-7})$ satisfies the Heegner hypothesis. The associated Heegner point $y_{K}=(-1,-1)$ satisfies Assumption $\star$. The set $\mathcal{S}$ consists of primes $\ell$ such that $\ell \equiv 1,2,4(\bmod 7)$ and $\ell \equiv 5$ $(\bmod 8)$ :

$$
\mathcal{S}=\{29,37,53,109,149,197,277,317,373,389, \ldots,\} .
$$

By Theorem 1.4, we have

$$
r_{\text {an }}\left(E^{( \pm \ell)} / K\right)=1, \text { for any } \ell \in \mathcal{S} .
$$

We compute the global root number $w\left(E^{( \pm \ell)} / \mathbb{Q}\right)=-1$ and conclude that

$$
r_{\text {an }}\left(E^{( \pm \ell)} / \mathbb{Q}\right)=1, \quad r_{\text {an }}\left(E^{( \pm 7 \ell)} / \mathbb{Q}\right)=0, \text { for any } \ell \in \mathcal{S} .
$$

Remark 2.2. Notice the two congruence conditions for $\ell \in S$ are both necessary for the conclusion: for example, we have $r_{\text {an }}\left(E^{(\ell)}\right)=2$ for $\ell=31$ and $r_{\text {an }}\left(E^{(7 \ell)}\right)=2$ for $\ell=5$.

Example 2.3. Consider the elliptic curve

$$
E=256 a 1: y^{2}=x^{3}+x^{2}-3 x+1
$$

with $E(\mathbb{Q})[2] \cong \mathbb{Z} / 2 \mathbb{Z}$. It has $j$-invariant 8000 and $\mathrm{CM}$ by $\mathbb{Q}(\sqrt{-2})$. The imaginary quadratic field $K=\mathbb{Q}(\sqrt{-7})$ satisfies the Heegner hypothesis. The associated Heegner point $y_{K}=(0,1)$ satisfies Assumption ( $₫$. The 2-isogenous curve is

$$
E_{0}=256 a 2: y^{2}=x^{3}+x^{2}-13 x-21 .
$$

We have $\mathbb{Q}(E[2])=\mathbb{Q}\left(E_{0}[2]\right)=\mathbb{Q}(\sqrt{2})$ and $\mathbb{Q}(\sqrt{-N})=\mathbb{Q}(i)$. Hence $\mathbb{Q}\left(E[2], E_{0}[2]\right)$ and $\mathbb{Q}(\sqrt{-N})$ are linearly disjoint. Since there is no odd prime $q \| N$, Theorem 1.7 implies that Silverman's conjecture holds for $E$.

In fact, the set $\mathcal{S}$ in this case consists of primes $\ell$ such that $\ell \equiv 1,2,4(\bmod 7)$ and $\ell \equiv 3,5$ $(\bmod 8)$ :

$$
\mathcal{S}=\{11,29,37,43,53,67,107,109,149,163,179,197,211,277,317,331, \ldots\} .
$$

Computing the global root number gives

$$
r_{\text {an }}\left(E^{(\ell)} / \mathbb{Q}\right)=1, \quad r_{\text {an }}\left(E^{(-\ell)} / \mathbb{Q}\right)=0, \text { for any } \ell \in \mathcal{S} .
$$

\section{Proof of Theorem 1.4}

3.1. Congruences between Heegner points. We first recall the main theorem of [KL16].

Theorem 3.1. Let $E$ and $E^{\prime}$ be two elliptic curves over $\mathbb{Q}$ of conductors $N$ and $N^{\prime}$ respectively. Suppose $p$ is a prime such that there is an isomorphism of semisimplified $G_{\mathbb{Q}}:=\operatorname{Gal}(\overline{\mathbb{Q}} / \mathbb{Q})$ representations

$$
E\left[p^{m}\right]^{\mathrm{ss}} \cong E^{\prime}\left[p^{m}\right]^{\mathrm{ss}}
$$


for some $m \geq 1$. Let $K$ be an imaginary quadratic field satisfying the Heegner hypothesis for both $N$ and $N^{\prime}$. Let $P \in E(K)$ and $P^{\prime} \in E^{\prime}(K)$ be the Heegner points. Assume $p$ is split in $K$. Then we have

$$
\left(\prod_{\ell \mid p N N^{\prime} / M} \frac{\left|\tilde{E}^{\mathrm{ns}}\left(\mathbb{F}_{\ell}\right)\right|}{\ell}\right) \cdot \log _{\omega_{E}} P \equiv \pm\left(\prod_{\ell \mid p N N^{\prime} / M} \frac{\left|\tilde{E}^{\prime, \mathrm{ns}}\left(\mathbb{F}_{\ell}\right)\right|}{\ell}\right) \cdot \log _{\omega_{E^{\prime}}} P^{\prime} \quad\left(\bmod p^{m}\right) .
$$

Here

$$
M=\prod_{\substack{\ell \mid \operatorname{gcd}\left(N, N^{\prime}\right) \\ a_{\ell}(E) \equiv a_{\ell}\left(E^{\prime}\right)}} \ell^{\operatorname{ord}_{\ell}\left(N N^{\prime}\right)} .
$$

3.2. Proof of Theorem 1.4. For a prime $\ell \nmid N d$, we have $a_{\ell}(E)= \pm a_{\ell}\left(E^{(d)}\right)$ since $E^{(d)}$ is a quadratic twist of $E$. Since $E(\mathbb{Q})[2] \neq 0$, we know that $\left|E\left(\mathbb{F}_{\ell}\right)\right|$ and $\left|E^{(d)}\left(\mathbb{F}_{\ell}\right)\right|$ are even since the reduction $\bmod \ell$ map is injective on prime-to- $\ell$ torsion. Hence if $\ell \neq 2$, then $a_{\ell}(E), a_{\ell}\left(E^{(d)}\right)$ are also even. Since $a_{\ell}(E)= \pm a_{\ell}\left(E^{(d)}\right)$, we obtain the following mod 4 congruence

$$
a_{\ell}(E) \equiv a_{\ell}\left(E^{(d)}\right) \quad(\bmod 4), \quad \text { for any } \ell \nmid 2 N d .
$$

It follows that we have an isomorphism of $G_{\mathbb{Q}}$-representations

$$
E[4]^{\mathrm{ss}} \cong E^{(d)}[4]^{\mathrm{ss}} .
$$

Now we can apply Theorem 3.1 to $E^{\prime}=E^{(d)}, p=2$ and $m=2$. By assumption, any prime $\ell \mid 2 N$ splits in $K$. By the definition of $\mathcal{S}$, the prime $\ell=|d|$ splits in $K$. Notice the odd prime factors of $N^{\prime}=N\left(E^{(d)}\right)$ are exactly the odd prime factors of $N d$, thus $K$ also satisfies the Heegner hypothesis for $N^{\prime}$.

Let $\ell \mid \operatorname{gcd}\left(N, N^{\prime}\right)$ be an odd prime. We have:

(1) if $\ell \| N$, then $a_{\ell}(E), a_{\ell}\left(E^{(d)}\right) \in\{ \pm 1\}$ is determined by their local root numbers at $\ell$. By the definition of $\mathcal{N}$, we know that $\ell$ splits in $\mathbb{Q}(\sqrt{d})$, and hence $E / \mathbb{Q}_{\ell}$ and $E^{(d)} / \mathbb{Q}_{\ell}$ are isomorphic. It follows that $a_{\ell}(E)=a_{\ell}\left(E^{(d)}\right)$.

(2) if $\ell^{2} \mid N$, then $a_{\ell}(E)=a_{\ell}\left(E^{(d)}\right)=0$,

Therefore $M$ is divisible by all the prime factors of $\operatorname{gcd}\left(N, N^{\prime}\right)$. Notice the odd part of $\operatorname{gcd}\left(N, N^{\prime}\right)$ equals to the odd part of $N$, so the congruence formula in Theorem 3.1 implies

$$
\prod_{\ell \mid 2 d} \frac{\left|\tilde{E}^{\mathrm{ns}}\left(\mathbb{F}_{\ell}\right)\right|}{\ell} \cdot \log _{\omega_{E}} P \equiv \pm \prod_{\ell \mid 2 d} \frac{\left|\tilde{E}^{(d), \mathrm{ns}}\left(\mathbb{F}_{\ell}\right)\right|}{\ell} \cdot \log _{\omega_{E}(d)} P^{(d)} \quad(\bmod 4) .
$$

For $\ell=|d|$, we have

$$
\left|E\left(\mathbb{F}_{\ell}\right)\right| \not \equiv 0 \quad(\bmod 4)
$$

by the definition of $\mathcal{S}$. Now Assumption $(\star x)$ implies that the left-hand-side of (1) is nonzero mod 4. Hence the right-hand-side of (1) is also nonzero. In particular, the Heegner point $P^{(d)} \in E^{(d)}(K)$ is non-torsion, and hence $r_{\text {an }}\left(E^{(d)} / K\right)=1$ by the theorem of Gross-Zagier [GZ86] and Kolyvagin Kol90, Kol88, as desired. 


\section{Proof of Theorem 1.7}

4.1. Elliptic curves with partial 2-torsion and no rational cyclic 4-isogeny. Let $E$ be an elliptic curve of conductor $N$. Assume $E(\mathbb{Q})[2] \cong \mathbb{Z} / 2 \mathbb{Z}$. Then $\mathbb{Q}(E[2]) / \mathbb{Q}$ is the quadratic extension $\mathbb{Q}\left(\sqrt{\Delta_{E}}\right)$, where $\Delta_{E}$ is the discriminant of a Weierstrass equation of $E$.

Let $\phi: E \rightarrow E_{0}:=E / E(\mathbb{Q})[2]$ be the natural 2-isogeny. By [Kla17, Lem. 4.2 (i)], $E$ has no rational cyclic 4 -isogeny if and only if $\mathbb{Q}\left(E_{0}[2]\right) / \mathbb{Q}$ is a quadratic extension. Assume we are in this case, then $\mathbb{Q}\left(E_{0}[2]\right)=\mathbb{Q}\left(\sqrt{\Delta_{E_{0}}}\right)$.

Lemma 4.1. Let $\ell \nmid N$ be a prime. Then the following are equivalent:

(1) $\left|E\left(\mathbb{F}_{\ell}\right)\right| \not \equiv 0(\bmod 4)$,

(2) $E\left(\mathbb{F}_{\ell}\right)[2] \cong E_{0}\left(\mathbb{F}_{\ell}\right)[2] \cong \mathbb{Z} / 2 \mathbb{Z}$,

(3) $\ell$ is inert in both $\mathbb{Q}(E[2])$ and $\mathbb{Q}\left(E_{0}[2]\right)$.

Proof. Since $E$ and $E_{0}$ are isogenous and $\ell$ is a prime of good reduction, we know that $\left|E\left(\mathbb{F}_{\ell}\right)\right|=$ $\left|E_{0}\left(\mathbb{F}_{\ell}\right)\right|$. So $\left|E\left(\mathbb{F}_{\ell}\right)\right| \not \equiv 0(\bmod 4)$ if and only if $\left|E_{0}\left(\mathbb{F}_{\ell}\right)\right| \not \equiv 0(\bmod 4)$. In this case, certainly

(2) holds. Conversely, if (2) holds, then $E\left(\mathbb{F}_{\ell}\right)[4] \cong \mathbb{Z} / 2 \mathbb{Z}$ (otherwise $E\left(\mathbb{F}_{\ell}\right)[4] \cong \mathbb{Z} / 4 \mathbb{Z}$, and thus $E_{0}\left(\mathbb{F}_{\ell}\right)[2] \cong \mathbb{Z} / 2 \mathbb{Z} \times \mathbb{Z} / 2 \mathbb{Z}$ generated by $\phi\left(E\left(\mathbb{F}_{\ell}\right)[4]\right)$ and the kernel of the dual isogeny $\left.\hat{\phi}: E_{0} \rightarrow E\right)$, hence $\left|E\left(\mathbb{F}_{\ell}\right)\right| \not \equiv 0(\bmod 4)$. We have shown that $(1)$ is equivalent to $(2)$.

Moreover, $E\left(\mathbb{F}_{\ell}\right)[2] \cong \mathbb{Z} / 2 \mathbb{Z}$ (resp. $\mathbb{Z} / 2 \mathbb{Z} \times \mathbb{Z} / 2 \mathbb{Z}$ ) if and only if $\mathbb{Q}_{\ell}(E[2]) / \mathbb{Q}_{\ell}$ is a quadratic extension (resp. the trivial extension), if and only if $\ell$ is inert (resp. split) in $\mathbb{Q}(E[2])$. Similarly we know that $E_{0}\left(\mathbb{F}_{\ell}\right)[2] \cong \mathbb{Z} / 2 \mathbb{Z}$ if and only if $\ell$ is inert in $\mathbb{Q}\left(E_{0}[2]\right)$. It follows that $(2)$ is equivalent to (3).

4.2. Proof of Theorem 1.7. By assumption, the fields $\mathbb{Q}\left(E[2], E_{0}[2]\right), \mathbb{Q}(\sqrt{q})$ ( $q$ runs all odd prime $q \| N)$ are linearly disjoint. Since $K$ satisfies the Heegner hypothesis for $N$ and 2 splits in $K$, we know the discriminant $d_{K}$ of $K$ is coprime to $2 N$, hence $K$ is also linearly disjoint from the fields $\mathbb{Q}\left(E[2], E_{0}[2]\right)$ and $\mathbb{Q}(\sqrt{q})$ 's. It follows from Chebotarev's density that there is a positive density set $\mathcal{T}$ of primes $\ell \nmid 2 N$ such that

(1) $\ell$ is split in $K$,

(2) $\ell$ is inert in both $\mathbb{Q}(E[2])$ and $\mathbb{Q}\left(E_{0}[2]\right)$,

(3) $\ell$ is split in $\mathbb{Q}(\sqrt{q})$ for any odd prime $q \| N$.

By Lemma 4.1, we know $\mathcal{T} \subseteq \mathcal{S}$. For $\ell \in \mathcal{T}$, we consider $d=\ell^{*}:=(-1)^{(\ell-1) / 2} \ell$. By the quadratic reciprocity law, we know that odd $q \| N$ is split in $\mathbb{Q}\left(\sqrt{\ell^{*}}\right)$ if and only if $\ell$ is split in $\mathbb{Q}(\sqrt{q})$. In particular, for any $\ell \in \mathcal{T}$, we have $\ell^{*} \in \mathcal{N}$. Now Theorem 1.4 implies that $r_{\text {an }}\left(E^{\left(\ell^{*}\right)} / K\right)=1$. Moreover,

$$
r_{\text {an }}\left(E^{\left(\ell^{*}\right)} / \mathbb{Q}\right)= \begin{cases}0, & w\left(E^{\left(\ell^{*}\right)} / \mathbb{Q}\right)=+1, \\ 1, & w\left(E^{\left(\ell^{*}\right)} / \mathbb{Q}\right)=-1 .\end{cases}
$$

Since $\mathbb{Q}\left(\sqrt{\ell^{*}}\right)$ has discriminant coprime to $2 N$, we have the well known formula

$$
w\left(E^{\left(\ell^{*}\right)} / \mathbb{Q}\right)=w(E / \mathbb{Q}) \cdot\left(\frac{\ell^{*}}{-N}\right) .
$$

By the quadratic reciprocity law, we obtain

$$
w\left(E^{\left(\ell^{*}\right)} / \mathbb{Q}\right)=w(E / \mathbb{Q}) \cdot\left(\frac{-N}{\ell}\right) .
$$


By assumption, $\mathbb{Q}(\sqrt{-N})$ is also linearly disjoint from the fields considered above, hence the global root number $w\left(E^{\left(\ell^{*}\right)} / \mathbb{Q}\right)$ takes both signs for a positive proportion of $\ell \in \mathcal{T}$ by Chebotarev's density. Therefore $r_{\text {an }}\left(E^{\left(\ell^{*}\right)} / \mathbb{Q}\right)$ takes both values 0 and 1 for a positive proportion of $\ell \in \mathcal{T}$, as desired.

\section{REFERENCES}

[Bir70] B. J. Birch. Elliptic curves and modular functions. In Symposia Mathematica, Vol. IV (INDAM, Rome, 1968/69), pages 27-32. Academic Press, London, 1970.

[CLTZ15] John Coates, Yongxiong Li, Ye Tian, and Shuai Zhai. Quadratic twists of elliptic curves. Proc. Lond. Math. Soc. (3), 110(2):357-394, 2015.

[GZ86] Benedict H. Gross and Don B. Zagier. Heegner points and derivatives of L-series. Invent. Math., 84(2):225320, 1986.

[KL16] D. Kriz and C. Li. Congruences between Heegner points and quadratic twists of elliptic curves. ArXiv e-prints, June 2016.

[Kla17] Zev Klagsbrun. Selmer ranks of quadratic twists of elliptic curves with partial rational two-torsion. Trans. Amer. Math. Soc., 369(5):3355-3385, 2017.

[Kol88] V. A. Kolyvagin. Finiteness of $E(\mathbf{Q})$ and $\amalg(E, \mathbf{Q})$ for a subclass of Weil curves. Izv. Akad. Nauk SSSR Ser. Mat., 52(3):522-540, 670-671, 1988.

[Kol90] V. A. Kolyvagin. Euler systems. In The Grothendieck Festschrift, Vol. II, volume 87 of Progr. Math., pages 435-483. Birkhäuser Boston, Boston, MA, 1990.

[Mon90] Paul Monsky. Mock Heegner points and congruent numbers. Math. Z., 204(1):45-67, 1990.

[Ono97] K. Ono. Twists of elliptic curves. Compositio Math., 106(3):349-360, 1997.

[OS98] Ken Ono and Christopher Skinner. Non-vanishing of quadratic twists of modular $L$-functions. Invent. Math., 134(3):651-660, 1998.

[Sag16] The Sage Developers. SageMath, the Sage Mathematics Software System (Version 7.2), 2016. http://www . sagemath. org.

[Ste75] N. M. Stephens. Congruence properties of congruent numbers. Bull. London Math. Soc., 7:182-184, 1975.

E-mail address: dkriz@princeton.edu

Department of Mathematics, Princeton University, Fine Hall, Washington Rd, Princeton, NJ 08544

E-mail address: chaoli@math.columbia.edu

Department of Mathematics, Columbia University, 2990 Broadway, New York, Ny 10027 